\title{
CONVERGENCE OF DOUBLE SERIES OF RANDOM ELEMENTS IN BANACH SPACES
}

\author{
Nguyen Duy Tien and Le Van Dung
}

\begin{abstract}
For a double array of random elements $\left\{X_{m n} ; m \geq 1, n \geq 1\right\}$ in a $p$-uniformly smooth Banach space, $\left\{b_{m n} ; m \geq 1, n \geq 1\right\}$ is an array of positive numbers, convergence of double random series $\sum_{m=1}^{\infty} \sum_{n=1}^{\infty} X_{m n}$, $\sum_{m=1}^{\infty} \sum_{n=1}^{\infty} b_{m n}^{-1} X_{m n}$ and strong law of large numbers

$$
b_{m n}^{-1} \sum_{i=1}^{m} \sum_{j=1}^{n} X_{i j} \rightarrow 0 \text { as } m \wedge n \rightarrow \infty
$$
\end{abstract}

are established.

\section{Introduction}

Consider a double array $\left\{X_{m n} ; m \geq 1, n \geq 1\right\}$ of random elements defined on a probability space $(\Omega, \mathcal{F}, P)$ taking values in a real separable Banach space $\mathcal{X}$ with norm $\|\cdot\|,\left\{b_{m n} ; m \geq 1, n \geq 1\right\}$ is an array of positive numbers. In the current work, we establish convergence a.s of double random series $\sum_{m=1}^{\infty} \sum_{n=1}^{\infty} X_{m n}$ and $\sum_{m=1}^{\infty} \sum_{n=1}^{\infty} b_{m n}^{-1} X_{m n}$, and since the convergence of double random series $\sum_{m=1}^{\infty} \sum_{n=1}^{\infty} b_{m n}^{-1} X_{m n}$ we obtain strong laws of large numbers $b_{m n}^{-1} \sum_{i=1}^{m} \sum_{j=1}^{n} X_{i j} \rightarrow 0$ as $m \wedge n \rightarrow \infty$.

Strong law of larger number for double array of random element in Banach spaces have studied by many authors. For example, Dung et al. [1], Dung and Tien [2], Quang et al. [8], Roralsky and Thanh [9], Stadtmuller and Thanh [11]. The three-series theorem for martingale in Banach spaces in case of single series was established by Tien [13]. However, convergence of double random series has not been studied. In this paper we not only extend some results of Su and Tong [12] and Hong and Tsay [4] but also establish the convergence of double random series.

Received May 20, 2011

2010 Mathematics Subject Classification. 60F15, 60B12.

Key words and phrases. convergence of double random series, strong laws of large numbers, $p$-uniformly smooth Banach spaces, double array of random elements.

This research has been partially supported by Vietnam's National Foundation for Science and Technology Development (NAFOSTED), grant no. 101.03-2010.06. 


\section{Preliminaries}

Technical definitions relevant to the current work will be discussed in this section.

For $a, b \in \mathbb{R}, \min \{a, b\}$ and $\max \{a, b\}$ will be denoted, respectively, by $a \wedge b$ and $a \vee b$. Denote $\mathbb{N}$ be the set of all positive integers, for $(i, j)$ and $(m, n) \in \mathbb{N}^{2}$, $(i, j) \prec(m, n)$ means that $i \leq m$ and $j \leq n$. Throughout this paper, the symbol $C$ will denote a generic constant $(0<C<\infty)$ which is not necessarily the same one in each appearance.

Scalora [10] introduced the idea of the conditional expectation of a random element in a Banach space. For a random element $V$ and sub $\sigma$-algebra $\mathcal{G}$ of $\mathcal{F}$, the conditional expectation $E(V \mid \mathcal{G})$ is defined analogously to that in the random variable case and enjoys similar properties.

A real separable Banach space $\mathcal{X}$ is said to be $p$-uniformly smooth $(1 \leq p \leq$ 2 ) if there exists a finite positive constant $C$ such that such that for any $L^{p}$ integrable $\mathcal{X}$-valued martingale difference sequence $\left\{X_{n}, n \geq 1\right\}$,

$$
E\left\|\sum_{i=1}^{n} X_{n}\right\|^{p} \leq C \sum_{i=1}^{n} E\left\|X_{i}\right\|^{p} .
$$

Clearly every real separable Banach space is of 1-uniformly smooth and the real line (the same as any Hilbert space) is of 2-uniformly smooth. If a real separable Banach space of $p$-uniformly smooth for some $1<p \leq 2$, then it is of $r$-uniformly smooth for all $r \in[1, p)$. For more details, the reader may refer to Pisier [7].

To prove the main result we need the following lemmas.

Lemma 2.1. Let $\left\{S_{m n} ; m \geq 1, n \geq 1\right\}$ be an array of random elements taking values in Banach space $\mathcal{X}$. Then, $S_{m n}$ converges a.s. as $m \wedge n \rightarrow \infty$ if only if for all $\varepsilon>0$,

$$
\lim _{N \rightarrow \infty} P\left(\sup _{\substack{N \leq m \leq p \\ N \leq n \leq q}}\left\|S_{p q}-S_{m n}\right\|>\varepsilon\right)=0 .
$$

Proof. Omitted.

Remark 2.2. Since inequalities

$$
\sup _{\substack{m \leq p \\ n \leq q}}\left\|S_{p q}-S_{m n}\right\| \leq \sup _{\substack{m \wedge n \leq p^{\prime} \leq p \\ m \wedge n \leq q^{\prime} \leq q}}\left\|S_{p^{\prime} q^{\prime}}-S_{p q}\right\| \leq 2 \sup _{\substack{m \leq p \\ n \leq q}}\left\|S_{p q}-S_{m n}\right\|,
$$

we have that the condition (2.1) is equivalent with

$$
\lim _{m \wedge n \rightarrow \infty} P\left(\sup _{\substack{m \leq p \\ n \leq q}}\left\|S_{p q}-S_{m n}\right\|>\varepsilon\right)=0 .
$$


Lemma 2.3. Let $\left\{a_{m n i j} ; 1 \leq i \leq m, 1 \leq j \leq n\right\}$ be an array of positive constants such that

$$
\sup _{m \geq 1, n \geq 1} \sum_{i=1}^{m} \sum_{j=1}^{n} a_{m n i j} \leq C<\infty \text { and } \lim _{m \wedge n \rightarrow \infty} a_{m n i j}=0 \text { for fixed } i, j .
$$

If $\left\{x_{m n} ; m \geq 1, n \geq 1\right\}$ is a double array of positive real numbers satisfying

$$
\lim _{m \vee n \rightarrow \infty} x_{m n}=0,
$$

then

$$
\lim _{m \wedge n \rightarrow \infty} \sum_{i=1}^{m} \sum_{j=1}^{n} a_{m n i j} x_{i j}=0 .
$$

Proof. For proof is similar that of Lemma 2.2 of Stadtmuller and Thanh [11].

Lemma $2.4([1])$. Let $1 \leq p \leq 2$. Let $\left\{X_{i j} ; 1 \leq i \leq m, 1 \leq j \leq n\right\}$ be a collection of $m n$ random elements in a real separable Banach space p-uniformly smooth $\mathcal{X}$. Set $\mathcal{F}_{i j}$ is a $\sigma$-algebra generated by the family of random elements $\left\{X_{k l} ; k<i\right.$ or $\left.l<j\right\}$ and $\mathcal{F}_{1,1}=\{\emptyset ; \Omega\}$. If $E\left(X_{i j} \mid \mathcal{F}_{i j}\right)=0$ for all $(i, j) \prec$ $(m, n)$, then

$$
E \max _{\substack{1 \leq k \leq m \\ 1 \leq l \leq n}}\left\|\sum_{i=1}^{k} \sum_{j=1}^{l} X_{i j}\right\|^{p} \leq C \sum_{i=1}^{m} \sum_{j=1}^{n} E\left\|X_{i j}\right\|^{p}
$$

where the constant $C$ is independent of $m$ and $n$.

Let $\left\{b_{m n} ; m \geq 1, n \geq 1\right\}$ be an array of positive numbers. We define

$$
N(x)=\operatorname{card}\left\{(m, n): b_{m n} \leq x\right\}
$$

and suppose that $N(x)<\infty, \forall x>0$.

Now we define two other functions $L(x)$ and $R_{p}(x)$ which are little different from that of $\mathrm{Su}$ and Tong [12]:

$$
L(x)=\int_{0}^{x} \frac{N(t) \log ^{+} N(t)}{t^{2}} d t \text { and } R_{p}(x)=\int_{x}^{\infty} \frac{N(t) \log ^{+} N(t)}{t^{p+1}} d t
$$

for $x>0$ and $p>0$. We have following lemma.

Lemma 2.5. Let $\left\{b_{m n} ; m \geq 1, n \geq 1\right\}$ be an array of positive numbers satisfying for each $m \geq 1$ and $n \geq 1, b_{i j} \leq b_{m n}$ for all $(i, j) \prec(m, n)$ and $b_{m n} \rightarrow \infty$ as $m \wedge n \rightarrow \infty$. Let $X$ be a non-negative real-valued random variables.

(i) If $\operatorname{EXL}(X)<\infty$, then

$$
\sum_{m=1}^{\infty} \sum_{n=1}^{\infty} P\left(X>b_{m n}\right)<\infty
$$


and

$$
\sum_{m=1}^{\infty} \sum_{n=1}^{\infty} \frac{1}{b_{m n}} \int_{b_{m n}}^{\infty} P(X>s) d s<\infty .
$$

(ii) If $E X^{p} R_{p}(X)<\infty$ for some $p>0$, then

$$
\sum_{m=1}^{\infty} \sum_{n=1}^{\infty} \frac{1}{b_{m n}^{p}} \int_{0}^{b_{m n}} s^{p-1} P(X>s) d s<\infty .
$$

Proof. First we prove (i). Suppose that $E X L(X)<\infty$, denote $d_{k}$ be the number of divisors of $k$ and noting that $N(x)$ is non-decreasing we have

$$
\begin{aligned}
\sum_{m=1}^{\infty} \sum_{n=1}^{\infty} P\left(X>b_{m n}\right) & \leq \sum_{m=1}^{\infty} \sum_{n=1}^{\infty} P\left(N(X)>N\left(b_{m n}\right)\right) \\
& \leq \sum_{m=1}^{\infty} \sum_{n=1}^{\infty} P(N(X)>m n) \\
& \leq \sum_{k=1}^{\infty} d_{k} P(N(X)>k) \\
& \leq C \sum_{k=1}^{\infty} \log (k) P(N(X)>k) \\
& \leq C \sum_{k=1}^{\infty}[(k+1) \log (k+1)-k \log (k)] P(N(X)>k) \\
& =C \sum_{k=1}^{\infty} k \log (k)[P(N(X) \leq k+1)-P(N(X) \leq k)] \\
& =C \sum_{k=1}^{\infty} k \log (k) \int_{k}^{k+1} d P(N(X) \leq x) \\
& \leq C \sum_{k=1}^{\infty} \int_{k}^{k+1} x \log x d P(N(X) \leq x) \\
& =C \int_{1}^{\infty} x \log x d P(N(X) \leq x) \\
& =C E N(X) \log +N(X) \leq C E X L(X)<\infty .
\end{aligned}
$$

Next we prove (2.3). Let $s=b_{m n} t$. Then we have

$$
\begin{aligned}
\sum_{m=1}^{k} \sum_{n=1}^{l} \frac{1}{b_{m n}} \int_{b_{m n}}^{\infty} P(X>s) d s & =\sum_{m=1}^{k} \sum_{n=1}^{l} \int_{1}^{\infty} P\left(\frac{X}{t}>b_{m n}\right) d t \\
& =\int_{1}^{\infty} \sum_{m=1}^{k} \sum_{n=1}^{l} P\left(\frac{X}{t}>b_{m n}\right) d t
\end{aligned}
$$




$$
\begin{aligned}
& \leq \int_{1}^{\infty} \sum_{m=1}^{\infty} \sum_{n=1}^{\infty} P\left(\frac{X}{t}>b_{m n}\right) d t \\
& \leq \int_{1}^{\infty} E N\left(\frac{X}{t}\right) \log ^{+} N\left(\frac{X}{t}\right) d t \\
& =\int_{0}^{\infty}\left(\int_{1}^{x} N\left(\frac{x}{t}\right) \log ^{+} N\left(\frac{x}{t}\right) d t\right) d P(X \leq x) \\
& =\int_{0}^{\infty} x\left(\int_{1}^{x} \frac{N(y) \log ^{+} N(y)}{y^{2}} d y\right) d P(X \leq x) \\
& =\operatorname{EXL}(X)<\infty .
\end{aligned}
$$

Letting $k \wedge l \rightarrow \infty$ we obtain (2.3).

Finally, we easily prove (ii) by using method of the proof is similar to that of $(2.3)$.

The array of random elements $\left\{X_{m n} ; m \geq 1, n \geq 1\right\}$ is said to be weakly mean dominated by the random element $X$ if, for some $0<C<\infty$,

$$
P\left\{\left\|X_{m n}\right\| \geq x\right\} \leq C P\{\|X\| \geq x\}
$$

for all $m \geq 1, n \geq 1$ and $x>0$.

\section{Main results}

With the preliminaries accounted for, the main results may now be established. In the following we let $\left\{X_{m n} ; m \geq 1, n \geq 1\right\}$ be an array of random elements defined on a probability $(\Omega, \mathcal{F}, P)$ and taking values in a real separable Banach space $\mathcal{X}$ with norm $\|\cdot\|, \mathcal{F}_{k l}$ be a $\sigma$-algebra generated by $\left\{X_{i j} ; i<k\right.$ or $\left.j<l\right\}, \mathcal{F}_{1,1}=\{\emptyset ; \Omega\}$. Suppose that $E\left(X_{m n} \mid \mathcal{F}_{m n}\right)=0$ for all $m \geq 1, n \geq 1$.

Theorem 3.1. Let $\mathcal{X}$ be a p-uniformly smooth Banach space for some $1 \leq p \leq$ 2. If

$$
\sum_{m=1}^{\infty} \sum_{n=1}^{\infty} E\left\|X_{m n}\right\|^{p}<\infty
$$

then

$$
\begin{aligned}
& \sum_{m=1}^{\infty} \sum_{n=1}^{\infty} X_{m n} \text { converges a.s., } \\
& \sum_{n=1}^{\infty} X_{m n} \text { converges a.s. for every } m \geq 1 \text { and } \\
& \sum_{m=1}^{\infty} X_{m n} \text { converges a.s. and for every } n \geq 1 .
\end{aligned}
$$


Proof. Set $S_{m n}=\sum_{i=1}^{m} \sum_{j=1}^{n} X_{i j}$.

For an arbitrary $\varepsilon>0$,

$$
\begin{aligned}
P\left(\max _{\substack{m \leq p \leq k \\
n \leq q \leq l}}\left\|S_{p q}-S_{m n}\right\|>\varepsilon\right) \leq & P\left(\max _{\substack{1 \leq m \leq k \\
n \leq q \leq l}}\left\|\sum_{i=1}^{m} \sum_{j=n}^{q} X_{i j}\right\|>\varepsilon / 2\right) \\
& +P\left(\max _{\substack{m \leq p \leq k \\
1 \leq n \leq l}}\left\|\sum_{i=1}^{m} \sum_{j=n}^{q} X_{i j}\right\|>\varepsilon / 2\right) .
\end{aligned}
$$

If $\mathcal{G}_{m q}$ is the $\sigma$-algebra generated by the family of random elements $\left\{X_{i j} ;(1 \leq\right.$ $i \leq k$ and $n \leq j<q)$ or $(1 \leq i<m$ and $n \leq j \leq k)\}$ for $1 \leq m \leq k$ and $n \leq q \leq l, \mathcal{G}_{1 n}=\{\emptyset ; \Omega\}$, then $\mathcal{G}_{m q} \subset \mathcal{F}_{m q}$ for all $1 \leq m \leq k, n \leq q \leq l$, which imply that $E\left(X_{m q} \mid \mathcal{G}_{m q}\right)=0$ for all $1 \leq m \leq k, n \leq q \leq l$.

Applying Markov inequality and Lemma 2.3 we obtain

$$
\begin{aligned}
P\left(\max _{\substack{1 \leq m \leq k \\
n \leq q \leq l}}\left\|\sum_{i=1}^{m} \sum_{j=n}^{q} X_{i j}\right\|>\varepsilon / 2\right) & \leq \frac{2^{p}}{\varepsilon^{p}} E\left(\max _{\substack{1 \leq m \leq k \\
n \leq q \leq l}}\left\|\sum_{i=1}^{m} \sum_{j=n}^{q} X_{i j}\right\|^{p}\right) \\
& \leq \frac{C}{\varepsilon^{p}} \sum_{i=1}^{k} \sum_{j=n}^{l} E\left\|X_{i j}\right\|^{p} .
\end{aligned}
$$

It is the same (3.6) we also have

$$
P\left(\max _{\substack{m \leq p \leq k \\ 1 \leq q \leq l}}\left\|\sum_{i=1}^{m} \sum_{j=n}^{q} X_{i j}\right\|>\varepsilon / 2\right) \leq \frac{C}{\varepsilon^{p}} \sum_{i=m}^{k} \sum_{j=1}^{l} E\left\|X_{i j}\right\|^{p} .
$$

It follows from (3.5), (3.6) and (3.7) that

$$
P\left(\max _{\substack{m \leq p \leq k \\ n \leq q \leq l}}\left\|S_{p q}-S_{m n}\right\|>\varepsilon\right) \leq \frac{C}{\varepsilon^{p}} \sum_{i=1}^{k} \sum_{j=n}^{l} E\left\|X_{i j}\right\|^{p}+\frac{C}{\varepsilon^{p}} \sum_{i=m}^{k} \sum_{j=1}^{l} E\left\|X_{i j}\right\|^{p} .
$$

This implies, by letting $k \wedge l \rightarrow \infty$, that

$$
P\left(\sup _{\substack{m \leq p \\ n \leq q}}\left\|S_{p q}-S_{m n}\right\|>\varepsilon\right) \leq \frac{C}{\varepsilon^{p}} \sum_{i=1}^{\infty} \sum_{j=n}^{\infty} E\left\|X_{i j}\right\|^{p}+\frac{C}{\varepsilon^{p}} \sum_{i=m}^{\infty} \sum_{j=1}^{\infty} E\left\|X_{i j}\right\|^{p} .
$$

We have by (3.1) that

$$
\sum_{i=1}^{\infty} \sum_{j=n}^{\infty} E\left\|X_{i j}\right\|^{p} \rightarrow 0 \text { as } n \rightarrow \infty
$$

and

$$
\sum_{i=m}^{\infty} \sum_{j=1}^{\infty} E\left\|X_{i j}\right\|^{p} \rightarrow 0 \text { as } m \rightarrow \infty
$$


hence,

$$
P\left(\sup _{\substack{m \leq p \\ n \leq q}}\left\|S_{p q}-S_{m n}\right\|>\varepsilon\right) \rightarrow 0 \text { as } m \wedge n \rightarrow \infty,
$$

which implies $S_{m n}$ converges a.s. as $m \wedge n \rightarrow \infty$ (by Lemma 2.1).

We now prove (3.3). For each $m \geq 1$, set $\mathcal{H}_{m, 1}=\{\Omega ; \emptyset\}$ and $\mathcal{H}_{m n}$ is the $\sigma$-algebra generated by the family of random elements $\left\{X_{m j} ; 1 \leq j<n\right\}$ for $n \geq 1$, we have that $\left\{S_{n}^{m}=\sum_{j=1}^{n} X_{m j}, \mathcal{H}_{m n} ; n \geq 1\right\}$ is a martingale satisfying $\sum_{n=1}^{\infty} E\left\|S_{n+1}^{m}-S_{n}^{m}\right\|^{p}<\infty$ (by (3.1)). Applying Theorem 2.2 of Woyczyński [14] we obtain the conclusion (3.3).

For proof of (3.4) is similar to that of (3.3). The proof is completed.

Remark 3.2. Noting that (3.2), (3.3) and (3.4) imply $X_{m n} \rightarrow 0$ a.s. as $m \vee n \rightarrow$ $\infty$. Hence, under the condition (3.1) we obtain $\lim _{m \vee n \rightarrow \infty}\left\|X_{m n}\right\|=0$ a.s. This remark will be used in Theorem 3.4 and Theorem 3.6.

Theorem 3.1 can be applied to obtain a version of the three-series theorem for double random series.

Theorem 3.3. Let $\mathcal{X}$ be a p-uniformly smooth Banach space for some $1 \leq p \leq$ 2 and $c$ be a positive constant. Set $Y_{m n}=X_{m n} I\left(\left\|X_{m n}\right\|>c\right)$. Suppose that $E\left(Y_{i j} \mid \mathcal{F}_{i j}\right)$ is measurable with respect to $\mathcal{F}_{m n}$ for all $i \leq m$ or $j \leq n$. If

(i) $\sum_{m=1}^{\infty} \sum_{n=1}^{\infty} P\left(\left\|X_{m n}\right\|>c\right)<\infty$,

(ii) $\sum_{m=1}^{\infty} \sum_{n=1}^{\infty} E\left(Y_{m n} \mid \mathcal{F}_{m n}\right)$ converges a.s., and

(iii) $\sum_{m=1}^{\infty} \sum_{n=1}^{\infty} E \|\left(Y_{m n}-E\left(Y_{m n} \mid \mathcal{F}_{m n}\right) \|^{p}<\infty\right.$, then $\sum_{m=1}^{\infty} \sum_{n=1}^{\infty} X_{m n}$ converges a.s.

Proof. We have by (i) that

$$
\sum_{m=1}^{\infty} \sum_{n=1}^{\infty} P\left(X_{m n} \neq Y_{m n}\right) \leq \sum_{m=1}^{\infty} \sum_{n=1}^{\infty} P\left(\left\|X_{m n}\right\|>c\right)<\infty .
$$

By virtue of Borel-Cantelli lemma, we have

$$
P\left(X_{m n} \neq Y_{m n} \text { i.o. }\right)=0 .
$$

So, to prove theorem, it suffices to show

$$
\sum_{m=1}^{\infty} \sum_{n=1}^{\infty} Y_{m n} \text { converges a.s. }
$$

In view of Theorem 3.1, we have by (iii) that

$$
\sum_{m=1}^{\infty} \sum_{n=1}^{\infty}\left(Y_{m n}-E\left(Y_{m n} \mid \mathcal{F}_{m n}\right)\right) \text { converges a.s. }
$$

Combining (ii) and (3.9) yields (3.8) holds.

The proof is completed. 
The following theorem is a version of Theorem 4.2 of Su and Tong [12] for double arrays of random elements in $p$-uniformly smooth Banach spaces.

Theorem 3.4. Let $\mathcal{X}$ be a p-uniformly smooth Banach space for some $1 \leq$ $p \leq 2$ and let $\left\{b_{m n} ; m \geq 1, n \geq 1\right\}$ be an array of positive numbers satisfying for each $m \geq 1$ and $n \geq 1, b_{i j} \leq b_{m n}$ for all $(i, j) \prec(m, n)$ and $b_{m n} \rightarrow \infty$ as $m \wedge n \rightarrow \infty$. Suppose that Suppose that $E\left(Y_{i j} \mid \mathcal{F}_{i j}\right)$ is measurable with respect to $\mathcal{F}_{m n}$ for all $i \leq m$ or $j \leq n$. Set

$$
N(x)=\operatorname{card}\left\{(m, n): b_{m n} \leq x\right\} \forall x>0 .
$$

If $\left\{X_{m n} ; m \geq 1, n \geq 1\right\}$ is weakly mean dominated by random element $X$ such that

$$
E\left(\|X\|^{p} R_{p}(\|X\|)\right)<\infty
$$

and

$$
E(\|X\| L(\|X\|))<\infty
$$

then

$$
\sum_{m=1}^{\infty} \sum_{n=1}^{\infty} \frac{X_{m n}}{b_{m n}} \text { converges a.s. }
$$

And if $\left\{b_{m n} ; m \geq 1, n \geq 1\right\}$ is an array of positive numbers satisfying for each $m \geq 1$ and $n \geq 1, b_{i j}<b_{m n}$ for all $(i, j) \prec(m, n)$ and $(i, j) \neq(m, n)$, $b_{m n} \rightarrow \infty$ as $m \wedge n \rightarrow \infty$, then

$$
\lim _{m \wedge n \rightarrow \infty} b_{m n}^{-1} \sum_{i=1}^{m} \sum_{j=1}^{n} X_{i j}=0 \quad \text { a.s. }
$$

Proof. For each $m, n$, set $Y_{m n}=X_{m n} I\left(\left\|X_{m n}\right\| \leq b_{m n}\right), Z_{m n}=X_{m n} I\left(\left\|X_{m n}\right\|\right.$ $\left.>b_{m n}\right), U_{m n}=Y_{m n}-E\left(Y_{m n} \mid \mathcal{F}_{m n}\right), V_{m n}=Z_{m n}-E\left(Z_{m n} \mid \mathcal{F}_{m n}\right)$. It is clear that $X_{m n}=U_{m n}+V_{m n}$. Moreover, $E\left(U_{m n} \mid \mathcal{F}_{m n}\right)=E\left(V_{m n} \mid \mathcal{F}_{m n}\right)=0$ for $m \geq 1$, $n \geq 1$. If $\mathcal{G}_{k l}^{\prime}$ and $\mathcal{G}_{k l}^{\prime \prime}$ are the $\sigma$-algebras generated by the family of random elements $\left\{U_{i j}: i<k\right.$ or $\left.j<l\right\}$ and $\left\{V_{l}: i<k\right.$ or $\left.j<l\right\}$, respectively, then $\mathcal{G}_{k l}^{\prime} \subset \mathcal{F}_{k l}$ and $\mathcal{G}_{k l}^{\prime \prime} \subset \mathcal{F}_{k l}$ for all $(k, l) \prec(m, n)$, which imply that $E\left(U_{k l} \mid \mathcal{G}_{k l}^{\prime}\right)=$ $E\left(V_{k l} \mid \mathcal{G}_{k l}^{\prime \prime}\right)=0$ for all $(k, l) \prec(m, n)$. Hence, in order to prove (3.12) we prove

$$
\sum_{m=1}^{\infty} \sum_{n=1}^{\infty} \frac{U_{m n}}{b_{m n}} \text { and } \sum_{m=1}^{\infty} \sum_{n=1}^{\infty} \frac{V_{m n}}{b_{m n}} \text { converge a.s. }
$$

Applying the strangle inequality and inequality (1.6) of Lemma 1.2 [3] we have

$$
\begin{aligned}
\sum_{m=1}^{\infty} \sum_{n=1}^{\infty} \frac{E\left\|V_{m n}\right\|}{b_{m n}} & \leq 2 \sum_{m=1}^{\infty} \sum_{n=1}^{\infty} \frac{E\left\|Z_{m n}\right\|}{b_{m n}} \\
& \leq 2 \sum_{m=1}^{\infty} \sum_{n=1}^{\infty} \frac{1}{b_{m n}} \int_{b_{m n}}^{\infty} P\left(\left\|X_{m n}\right\|>s\right) d s
\end{aligned}
$$




$$
\begin{aligned}
&+2 \sum_{m=1}^{\infty} \sum_{n=1}^{\infty} P\left(\left\|X_{m n}\right\|>b_{m n}\right) \\
& \leq C \sum_{m=1}^{\infty} \sum_{n=1}^{\infty} \frac{1}{b_{m n}} \int_{b_{m n}}^{\infty} P(\|X\|>s) d s \\
&+C \sum_{m=1}^{\infty} \sum_{n=1}^{\infty} P\left(\|X\|>b_{m n}\right) \\
&<\infty \text { (by Lemma 2.4) }
\end{aligned}
$$

which implies by Theorem 3.1 that

$$
\sum_{m=1}^{\infty} \sum_{n=1}^{\infty} \frac{V_{m n}}{b_{m n}} \text { converges a.s. }
$$

Again applying the strangle inequality and equality (1.5) of Lemma 1.2 [3] we have

$$
\begin{aligned}
\sum_{m=1}^{\infty} \sum_{n=1}^{\infty} \frac{E\left\|U_{m n}\right\|^{p}}{b_{m n}^{p}} \leq & C \sum_{m=1}^{\infty} \sum_{n=1}^{\infty} \frac{E\left\|Y_{m n}\right\|^{p}}{b_{m n}^{p}} \\
= & C \sum_{m=1}^{\infty} \sum_{n=1}^{\infty} \frac{1}{b_{m n}^{p}} \int_{b_{m n}}^{\infty} s^{p-1} P\left(\left\|X_{m n}\right\|>s\right) d s \\
& -C \sum_{m=1}^{\infty} \sum_{n=1}^{\infty} P\left(\left\|X_{m n}\right\|>b_{m n}\right) \\
\leq & C \sum_{m=1}^{\infty} \sum_{n=1}^{\infty} \frac{1}{b_{m n}^{p}} \int_{b_{m n}}^{\infty} s^{p-1} P(\|X\|>s) d s \\
& -C \sum_{m=1}^{\infty} \sum_{n=1}^{\infty} P\left(\|X\|>b_{m n}\right) \\
< & \infty(\text { by Lemma } 2.4)
\end{aligned}
$$

which implies by Theorem 3.1 that

$$
\sum_{m=1}^{\infty} \sum_{n=1}^{\infty} \frac{U_{m n}}{b_{m n}} \text { converges a.s. }
$$

Now we prove (3.13). Since (3.14) and (3.15) we have by Theorem 3.1 that $b_{m n}^{-1} V_{m n} \rightarrow 0$ a.s. and $b_{m n}^{-1} U_{m n} \rightarrow 0$ a.s. as $m \vee n \rightarrow \infty$. Hence,

$$
\lim _{m \vee n \rightarrow \infty} b_{m n}^{-1}\left\|X_{m n}\right\|=0 \text { a.s. }
$$

Applying Lemma 2.2 with $a_{m n i j}=\frac{b_{i j}}{b_{m n}}$ we have

$$
\lim _{m \wedge n \rightarrow \infty} b_{m n}^{-1} \sum_{i=1}^{m} \sum_{j=1}^{n}\left\|X_{i j}\right\| \rightarrow 0 \text { a.s. }
$$


and using the strangle inequality

$$
\left\|b_{m n}^{-1} \sum_{i=1}^{m} \sum_{j=1}^{n} X_{i j}\right\| \leq b_{m n}^{-1} \sum_{i=1}^{m} \sum_{j=1}^{n}\left\|X_{i j}\right\|
$$

we obtain (3.13).

Corollary 3.5. Let $\mathcal{X}$ be a p-uniformly smooth Banach space for some $1 \leq p \leq$ 2. Let $\left\{a_{m n} ; m \geq 1, n \geq 1\right\}$ be an array of real numbers such that $a_{m n} \neq 0$, let $\left\{b_{m n} ; m \geq 1, n \geq 1\right\}$ be an array of positive numbers satisfying for each $m \geq 1$ and $n \geq 1, b_{i j}<b_{m n}$ and $b_{i j} /\left|a_{i j}\right|<b_{m n} /\left|a_{m n}\right|$ for all $(i, j) \prec(m, n)$ and $(i, j) \neq(m, n), b_{m n} /\left|a_{m n}\right| \rightarrow \infty$ as $m \wedge n \rightarrow \infty$. Suppose that $E\left(X_{i j} I\left(\left\|X_{i j}\right\| \leq\right.\right.$ $\left.\left.b_{i j}\right) \mid \mathcal{F}_{i j}\right)$ is measurable with respect to $\mathcal{F}_{m n}$ for all $i \leq m$ or $j \leq n$. Set

$$
N(x)=\operatorname{card}\left\{(m, n): \frac{b_{m n}}{\left|a_{m n}\right|} \leq x\right\} \forall x>0 .
$$

If $\left\{X_{m n} ; m \geq 1, n \geq 1\right\}$ is weakly mean dominated by random element $X$ such that (3.10) and (3.11) hold, then

$$
\lim _{m \wedge n \rightarrow \infty} b_{m n}^{-1} \sum_{i=1}^{m} \sum_{j=1}^{n} a_{i j} X_{i j}=0 \quad \text { a.s. }
$$

Finally, we extend Theorem 2.1 of Hong and Tsay [4] to double array of random elements. It is the same Theorem 3.4, we establish convergence of double random series before obtaining strong laws of large numbers.

Theorem 3.6. Let $\mathcal{X}$ be a p-uniformly smooth Banach space for some $1 \leq$ $p \leq 2$ and let $\left\{b_{m n} ; m \geq 1, n \geq 1\right\}$ be an array of positive numbers. Suppose that $E\left(Y_{i j} \mid \mathcal{F}_{i j}\right)$ is measurable with respect to $\mathcal{F}_{m n}$ for all $i \leq m$ or $j \leq n$. Let $\left\{\Phi_{m n} ; m \geq 1, n \geq 1\right\}$ be an array of positive Borel functions and let $C_{m n} \geq 1$, $D_{m n} \geq 1, b_{m n} \geq 1,0<\beta_{m n} \leq p$ be constants satisfying for $u \geq v>0$,

$$
C_{m n} \frac{u^{b_{m n}}}{v^{b_{m n}}} \leq \frac{\Phi_{m n}(u)}{\Phi_{m n}(v)} \leq D_{m n} \frac{u^{\beta_{m n}}}{v^{\beta_{m n}}}
$$

If

$$
\sum_{m=1}^{\infty} \sum_{n=1}^{\infty} A_{m n} \frac{E \Phi_{m n}\left(\left\|X_{m n}\right\|\right)}{\Phi_{m n}\left(b_{m n}\right)}<\infty,
$$

where $A_{m n}=\max \left\{\frac{1}{C_{m n}}, D_{m n}\right\}$, then (3.12) holds. And if $\left\{b_{m n} ; m \geq 1, n \geq 1\right\}$ is an array of positive numbers satisfying for each $m \geq 1$ and $n \geq 1, b_{i j} \leq b_{m n}$ for all $(i, j) \prec(m, n)$ and $b_{m n} \rightarrow \infty$ as $m \wedge n \rightarrow \infty$, then (3.13) holds.

Proof. Set the same $Y_{m n}, Z_{m n}, U_{m n}$ and $V_{m n}$ as in the proof of Theorem 3.4. It is similar to the proof of Theorem 3.4, we show that

$$
\sum_{m=1}^{\infty} \sum_{n=1}^{\infty} \frac{E\left\|V_{m n}\right\|}{b_{m n}}<\infty
$$


and

$$
\sum_{m=1}^{\infty} \sum_{n=1}^{\infty} E \frac{\left\|U_{m n}\right\|^{p}}{b_{m n}^{p}}<\infty
$$

First we prove (3.16).

$$
\begin{aligned}
\sum_{m=1}^{\infty} \sum_{n=1}^{\infty} \frac{E\left\|V_{m n}\right\|}{b_{m n}} & \leq 2 \sum_{m=1}^{\infty} \sum_{n=1}^{\infty} E \frac{\left\|Z_{m n}\right\|}{b_{m n}} \\
& \leq 2 \sum_{m=1}^{\infty} \sum_{n=1}^{\infty} E\left(\frac{\left\|Z_{m n}\right\|}{b_{m n}}\right)^{\alpha_{m n}} \\
& \leq 2 \sum_{m=1}^{\infty} \sum_{n=1}^{\infty} \frac{1}{C_{m n}} E \frac{\Phi_{m n}\left(\left\|Z_{m n}\right\|\right)}{\Phi_{m n}\left(b_{m n}\right)} \\
& \leq 2 \sum_{m=1}^{\infty} \sum_{n=1}^{\infty} A_{m n} E \frac{\Phi_{m n}\left(\left\|Z_{m n}\right\|\right)}{\Phi_{m n}\left(b_{m n}\right)} \\
& \leq 2 \sum_{m=1}^{\infty} \sum_{n=1}^{\infty} A_{m n} E \frac{\Phi_{m n}\left(\left\|X_{m n}\right\|\right)}{\Phi_{m n}\left(b_{m n}\right)}<\infty
\end{aligned}
$$

Finally we prove (3.17).

$$
\begin{aligned}
\sum_{m=1}^{\infty} \sum_{n=1}^{\infty} E \frac{\left\|U_{m n}\right\|^{p}}{b_{m n}^{p}} & \leq 2^{p} \sum_{m=1}^{\infty} \sum_{n=1}^{\infty} E \frac{\left\|Y_{m n}\right\|^{p}}{b_{m n}^{p}} \\
& \leq C \sum_{m=1}^{\infty} \sum_{n=1}^{\infty} E\left(\frac{\left\|Y_{m n}\right\|}{b_{m n}}\right)^{\beta_{m n}} \\
& \leq C \sum_{m=1}^{\infty} \sum_{n=1}^{\infty} D_{m n} E \frac{\Phi_{m n}\left(\left\|Y_{m n}\right\|\right)}{\Phi_{m n}\left(b_{m n}\right)} \\
& \leq C \sum_{m=1}^{\infty} \sum_{n=1}^{\infty} A_{m n} E \frac{\Phi_{m n}\left(\left\|Y_{m n}\right\|\right)}{\Phi_{m n}\left(b_{m n}\right)} \\
& \leq C \sum_{m=1}^{\infty} \sum_{n=1}^{\infty} A_{m n} E \frac{\Phi_{m n}\left(\left\|X_{m n}\right\|\right)}{\Phi_{m n}\left(b_{m n}\right)}<\infty
\end{aligned}
$$

The proof is completed.

\section{References}

[1] L. V. Dung, Th. Ngamkham, N. D. Tien and A. I. Volodin, Marcinkiewicz-Zygmund type law of large numbers for double arrays of random elements in Banach spaces, Lobachevskii J. Math. 30 (2009), no. 4, 337-346.

[2] L. V. Dung and N. D. Tien, Mean convergence theorems and weak laws of large numbers for double arrays of random elements in Banach spaces, Bull. Korean Math. Soc. $\mathbf{4 7}$ (2010), no. 3, 467-482. 
[3] S. Gan, On almost sure convergence of weighted sums of random element sequences, Acta Math. Sci. Ser. B Engl. Ed. 30 (2010), no. 4, 1021-1028.

[4] J. I. Hong and J. Tsay, A strong law of large numbers for random elements in Banach spaces, Southeast Asian Bull. Math. 34 (2010), no. 2, 257-264.

[5] D. Landers and L. Rogge, Laws of large numbers for pairwise independent uniformly integrable random variables, Math. Nachr. 130 (1987), 189-192.

[6] M. Ordóñez Cabrera, Convergence of weighted sums of random variables and uniform integrability concerning the weights, Collect. Math. 45 (1994), no. 2, 121-132.

[7] G. Pisier, Probabilistic methods in the geometry of Banach spaces, Probability and analysis (Varenna, 1985), 167241, Lecture Notes in Math., 1206, Springer, Berlin, 1986.

[8] N. V. Quang, L. V. Thanh, and N. D. Tien, Almost sure convergence for double arrays of block-wise $M$-dependent random elements in Banach spaces, Georgian Mathematical Journal 18 (2011), 777-800.

[9] A. Rosalsky and L. V. Thanh, Strong and weak laws of large numbers for double sums of independent random elements in Rademacher type p Banach spaces, Stoch. Anal. Appl. 24 (2006), no. 6, 1097-1117.

[10] F. S. Scalora, Abstract martingale convergence theorems, Pacific J. Math. 11 (1961), $347-374$.

[11] U. Stadtmulle and L. V. Thanh, On the strong limit theorems for double arrays of blockwise M-dependent random variables, Acta Math. Sin. (Engl. Ser.) 27 (2011), no. 10, 19231934.

[12] C. Su and T. J. Tong, Almost sure convergence of the general Jamison weighted sum of $\mathcal{B}$-valued random variables, Acta Math. Sin. (Engl. Ser.) 20 (2004), no. 1, 181-192.

[13] N. D. Tien, On Kolmogorov's three series theorem and mean square convergence of martingales in a Banach space, Theory Probab. Appl. 24 (1980), no. 4, 797-808.

[14] W. A. Woyczyński, Geometry and martingales in Banach spaces, ProbabilityWinter School (Proc. Fourth Winter School, Karpacz, 1975), pp. 229-275. Lecture Notes in Math., Vol. 472, Springer, Berlin, 1975.

Nguyen Duy Tien

FaCUlty of Mathematics

NATIONAL University OF HaNoI

334 Nguyen Trai, Hanoi, Vietnam

E-mail address: nduytien2006@yahoo.com

Le VAN DUnG

FaCUlty of Mathematics

DANANG University of Education

459 Ton Duc Thang, Danang, Vietnam

E-mail address: lvdunght@gmail.com 